\title{
Dorsolateral prefrontal cortex dysfunction in the major psychoses; symptom or disease specificity?
}

\author{
R J Dolan, C J Bench, P F Liddle, K J Friston, C D Frith, P M Grasby, R S J Frackowiak
}

MRC Cyclotron Unit, Hammersmith Hospital, London, UK R J Dolan C J Bench P F Liddle K J Friston C D Frith P M Grasby R S Frackowiak

Academic Department of Psychiatry, Royal Free Hospital and School of Medicine, London, UK R J Dolan

C J Bench

P M Grasby

National Hospital for

Neurology and Neurosurgery, London, UK R J Dolan R S Frackowiak

Psychology Department University College

London, UK C D Frith

Correspondence to: Dr Dolan, Academic Department of Psychiatry, Royal Free Hospital and School of Medicine, Pond School of Medicine, Pond
Street, London NW3 2 QG, Street,

Received 7 August 1992 and in final revised form 25 February 1993. Accepted 9 March 1993

\begin{abstract}
Neurophysiological deficits in the left dorsolateral prefrontal cortex (DLPFC) have been described in positron emission tomography studies of schizophrenia and depression. In schizophrenia and depression this deficit has been associated with the syndromes of psychomotor poverty and psychomotor retardation, respectively. Such findings lead to a prediction that DLPFC dysfunction is symptom rather than disease related. This prediction was empirically tested in a retrospective study that pooled data from 40 patients meeting research diagnostic criteria for depression and 30 patients meeting DSM-III R criteria for schizophrenia. The patients were categorised into those with and without poverty of speech, a symptom that is an observable manifestation of psychomotor impairment. The profile of regional cerebral blood flow (rCBF), measured in all subjects under resting conditions, was subsequently compared in these two groups. Patients with poverty of speech had significantly lower rCBF in the left DLFPC. This reduction of $\mathrm{rCBF}$ was independent of diagnosis. The findings support the view that the study of symptoms, or symptom clusters, can provide information additional to that of traditional diagnostic systems in the study of the major psychoses.
\end{abstract}

$(F$ Neurol Neurosurg Psychiatry 1993;56:1290-1294)

Studies of cerebral neurophysiology, using functional imaging techniques, indicate multifocal regional abnormalities in both schizophrenia and depression. The presence of multifocal deficits in both disorders is consistent with current conceptualisations of an anatomically distributed neural basis to higher cerebral functions. ${ }^{12}$ Among the range of functional deficits reported in schizophrenia and depression, the most robust finding has been that of 'hypofrontality'. ${ }^{34}$ Recent studies, particularly those using more refined data analytic techniques, have provided greater anatomical specificity to this finding with the demonstration of dysfunction in the left dorsolateral prefrontal cortex (DLPFC) in both schizophrenia and depression. ${ }^{56}$

The characterisation of the phenomenological and neuropsychological correlates of biological dysfunctions is an important, though often elusive, goal in neuropsychiatric research. Nevertheless PET studies have begun to suggest important and reproducible phenomenological correlates of DLPFC dysfunction. DeLisi et al reported significant correlations between relative hypofrontality and symptom ratings for emotional withdrawal, disorientation, distractability and helplessness/hopelessness. ${ }^{7}$ In a more recent study of chronic schizophrenic patients, left DLPFC dysfunction was associated with a syndrome of psychomotor poverty. ${ }^{8}$ In depression functional deficits in the left DLPFC have also been associated with a syndrome of psychomotor retardation. ${ }^{9}$

These findings suggest that there are features common to the pathophysiology of both depression and schizophrenia, and in particular, that diminished psychomotor activity is associated with hypofrontality in both conditions. In this investigation we pooled PET data from two large cohorts of patients diagnosed as suffering with either schizophrenia or depression. This enabled us to test the hypothesis that hypofrontality is associated with diminished psychomotor activity, irrespective of diagnosis. Poverty of speech provides a measure of diminished psychomotor activity that can be assessed in a manner not likely to be confounded by qualitative affective differences between depressed and schizophrenic patients. Poverty of speech in this study is defined as a "restriction in the amount of spontaneous speech, so that replies to questions tend to be brief, concrete and unelaborated". ${ }^{10}$ Furthermore, poverty of speech is highly correlated with syndromes of psychomotor poverty in schizophrenia ${ }^{11}$ and psychomotor retardation in depression. ${ }^{12} \mathrm{We}$ have therefore tested the specific hypothesis that poverty of speech is associated with decreased perfusion in the left DLPFC in depression and schizophrenia in a manner that is independent of diagnosis.

\section{Subjects and methods}

SUBJECTS

The mode of selection and clinical characteristics of both the depressed and schizophrenic patients have been described previously. ${ }^{68}$ In summary, 40 depressed patients were administered the Schedule for Schizophrenia and Affective Disorders (SADS-L) ${ }^{13}$ and all met the Research Diagnostic Criteria ${ }^{14}$ for Major Depressive Disorder and scored over 17 on the Hamilton Depression Rating Scale 
(HAM-D). ${ }^{15}$ Exclusion criteria included age greater than 75 years, a history of alcohol or substance abuse, significant previous or current medical illness, a score of over 4 on the Hachinski ischaemic scale ${ }^{16}$ or focal abnormality on CT or MRI scanning. Half of this patient sample were receiving medication.

The 30 schizophrenic patients were aged less than 55 years and all met the DSM-III-R criteria for schizophrenia. ${ }^{17}$ Symptomatically all the patients were in a persistently stable state. These patients were assessed according to a protocol specified in the Comprehensive Assessment of Symptoms and History, which includes the Scales for the Assessment of Negative Symptoms (SANS). ${ }^{10}$ All the schizophrenic patients were receiving neuroleptic medication with the mean daily dose, expressed in chlorpromazine equivalents, being 1465 (SD 1011) mg. All patients, schizophrenic and depressed, were rated for depression on a subjective measure of dysphoria derived from the SADS (item 234).

All patients gave informed consent. Ethical approval was obtained from all referring hospitals and locally from the Royal Postgraduate Medical School Research Ethics Committee. Permission to administer radioisotopes was obtained from the Administration of Radioactive Substances Advisory Committee of the United Kingdom.

\section{PET METHODS}

Regional cerebral blood flow was measured under resting conditions, in a quiet darkened room, with the subjects supine in the PET scanner (CTI model 931-08/12). Subjects were asked to close their eyes during the examination but no other instructions were given. ${ }^{15} \mathrm{Oxygen}$ in the form of $\mathrm{C}^{15} \mathrm{O}_{2}$ mixed with air was administered via an oxygen mask according to an established protocol. ${ }^{10} \mathrm{~A}$ single scan was acquired over the final 10 minute period of an 18 minute inhalation and arterial blood samples were taken via an indwelling $22 \mathrm{~g}$ catheter at 0,5 and $10 \mathrm{~min}-$ utes into the acquisition period. A Hanning filter with a cut off frequency of 0.5 was used in the reconstruction of the images giving a transaxial resolution of $8.5 \mathrm{~mm}$. All scans were performed on the same equipment using the same methods over a three year period up to June 1991 .

\section{IMAGE ANALYSIS}

Image analysis was performed using SPM software (MRC Cyclotron Unit, London, UK) on a SPARC 1 workstation (Sun Microsystems, Surrey, UK) using an interactive image analysis software package (ANALYZE, Biodynamic Research Unit, Mayo Clinic, USA). Calculations and image matrix manipulations were performed in PRO MATLAB (Mathworks, New York).

\section{STEREOTACTIC NORMALISATION}

Each reconstructed rCBF scan consisting of 15 primary transverse planes was interpolated to 43 planes to render the voxels approximately cubic. The data were then trans- formed into a standard stereotactic space. ${ }^{18} 19$ Such transformation of the data allows for voxel by voxel averaging of data across subjects. In the standard space 1 voxel represents $2 \times 2 \times 4 \mathrm{~mm}$ in the $\mathrm{x}, \mathrm{y}$ and $\mathrm{z}$ dimensions, respectively, allowing direct cross reference to the anatomical features of a standard stereotactic atlas. ${ }^{20} \mathrm{~A}$ Gaussian filter 10 pixels wide was applied to smooth each image to accommodate inter-subject differences in gyral and functional anatomy and to suppress high frequency noise in the images.

DETECTION OF SIGNIFICANT CHANGES IN RCBF Differences in global activity between subjects were removed by analysis of covariance on a voxel by voxel basis with global counts as covariate according to a method previously described. ${ }^{18}$ For each voxel in stereotactic space, in each experimental group, the analysis of covariance generated a conditionspecific adjusted mean rCBF value (normalised to $50 \mathrm{ml} / \mathrm{dl} / \mathrm{min}$ ) and an associated adjusted error variance. Group means were then compared using the $t$-statistic (SPM $\{\mathrm{t}\}$ ). The resulting set of $t$ values constitutes a statistical parametric map. ${ }^{21}$ The omnibus significance of the statistical parametric maps (SPM) was assessed for the whole data set by comparing the observed and expected distribution of the $t$-statistic under the null hypothesis of no treatment effect. The expected and observed number of $t$ values exceeding a given threshold $(p<0.001)$ was compared (using the Chi squared test of proportions) and if significant the location of all voxels with a $t$ value equal to or greater than the chosen threshold was noted. The resulting SPMs of $t$ values were displayed within the standard stereotactic space, plane by plane, and as projections on to renderings of the medial and lateral cortical surfaces of the brain.

\section{STRATEGY FOR DATA ANALYSIS}

The first step in the analysis was the identification of pixels where there was a significant difference in rCBF between patients with and without poverty of speech. For this purpose we identified two groups from the combined patient sample: those with definite poverty of speech (score for the SADS poverty of speech item of 1 , or score for the SANS poverty of speech item of 2 or more), and those without definite poverty of speech. Having identified the locus of greatest difference in rCBF between the two groups, analysis of variance was used to examine the contributions of poverty of speech and of diagnosis to variance in $\mathrm{rCBF}$ at that site, and to test the null hypothesis of no interaction between these independent contributions. Furthermore, in the light of the possibility that depressed mood is associated with decreased rCBF in the DLPFC (irrespective of diagnosis), analysis of covariance was performed to allow for variation in severity of depressed mood. For this purpose the SADS item score for dysphoria was used as a measure of depressed mood. 
Table 1 Coordinates of the pixels where the most significant differences in blood flow were identified. Co-ordinates refer to the stereotaxic atlas of Talairach and Tournoux. The CBF values are in units of ml/dl tissue/min, and have been adjusted for a global mean blood

flow of $50 \mathrm{ml} / \mathrm{dl} / \mathrm{min}$. The $Z$ score is a measure of the degree of significance of the difference and is the number of standard deviations from the mean $t$ value in the $(t)$ statistical map of the $t$ value for the most significant pixel in the plane

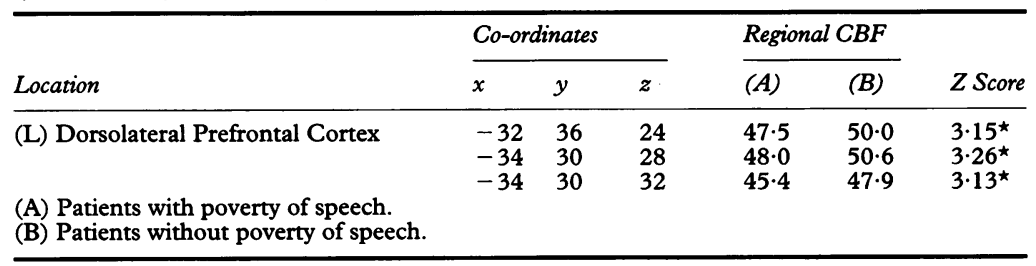

${ }^{\star} \mathrm{p}<0.001$.

\section{Results}

The mean (SD) age of the schizophrenic (25 male; 5 female) and depressed patients (25 male; 15 female) were 36 (10) and 57 (13), respectively. Twenty seven of the total sample of patients $(n=70)$ were classified as having poverty of speech. These comprised 15 patients with a diagnosis of depression and 12 with a diagnosis of schizophrenia. The mean (SD) ages [48 (15) vs 48 (16)] and sex distribution of the poverty (17 male; 10 female) and non-poverty (33 male; 10 female) of speech patients were not significantly different $\left(\chi^{2}=1 \cdot 54, \mathrm{~ns}\right)$.

POVERTY OF SPEECH VS NON POVERTY OF SPEECH PATIENTS

Using $\operatorname{SPM}\{t\}$ a categorical comparison was made of the rCBF profiles of poverty and nonpoverty of speech patients. There was a signifi- cant difference in the observed and expected distribution of $t$ values at a high threshold ( $p$ $<0.001)$. The SPM $\{t\}$ identified two regions where there was a significant relative decrease in $\mathrm{rCBF}$ in the poverty of speech group. The areas identified were centred on the left DLPFC (BA 46/9) and the left angular gyrus (BA 39) with the most significant differences centred on the left DLPFC. The $\operatorname{SPM}\{t\}$ is shown in the figure with the coordinates and rCBF values of maximal significance being displayed in table 1 .

The adjusted rCBF values, at the pixel coordinates of maximal difference in the above SPM $\{t\}$, were subsequently sampled for each individual subject. These values were entered into a two way analysis of variance (ANOVA), with diagnosis and poverty of speech as grouping variables. This analysis indicated a significant effect of poverty of speech on left DLPFC rCBF values $(p<0.005)$. A significant effect of diagnosis was also apparent with depressed patients displaying lower DLPFC rCBF values $(p<$ $0.01)$. No interaction effects were evident. However, as depressed mood has been shown to be independently associated with left DLPFC dysfunction its possible confounding effects were subsequently controlled by repeating the analysis with a rating of dysphoria as covariate. The results of this analysis (ANCOVA) are presented in table 2. After controlling for the effects of mood the only

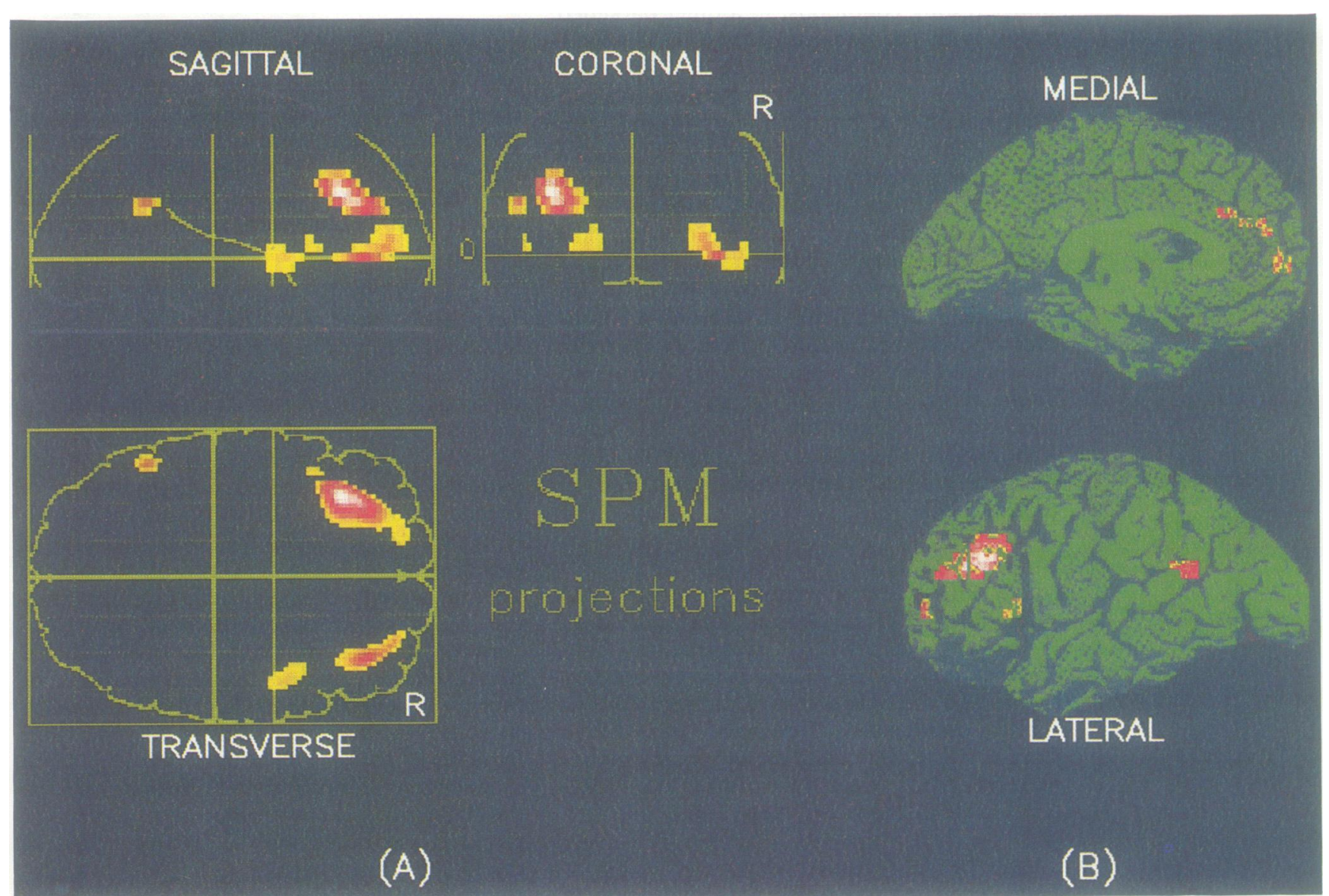

Figure Comparison of patients with $(n=27)$ and without $(n=43)$ poverty of speech using SPM $\{t\}$. Areas highlighted indicate regions where there are significant decreases $(p<0.01)$ in $r C B F$ in the poverty of speech relative to the non-poverty group. Regions where there are significant decreases are displayed as orthogonal projections within standard stereotactic space $(A)$ or rendered on to the medial and lateral cortical surface of the left hemisphere $(B)$. 
Table 2 Two way ANOVA, with dysphoria as covariate, of $r C B F$ values in the left dorsolateral prefrontal cortex in depressed and schizophrenic patients with and without poverty of speech

\begin{tabular}{llll}
\hline & $d f$ & $F$ & $p$ \\
\hline Diagnosis (schizophrenia v depression & 1 & $0 \cdot 34$ & $\mathrm{~ns}$ \\
Symptom (poverty of speech present v absent) & 1 & $9 \cdot 1$ & 0.005 \\
Interaction (diagnosis by poverty of speech) & 1 & $0 \cdot 12$ & $\mathrm{~ns}$ \\
\hline
\end{tabular}

significant effect was that of poverty of speech. Furthermore, there was no interaction effect between diagnosis and poverty of speech. The absence of an interaction indicates that the effects of poverty of speech on rCBF values in the DLPFC is independent of diagnosis. It also indicates that an effect of poverty of speech is present in both diagnostic groups.

\section{Discussion}

The present study provides evidence that, after allowing for variance due to depressed mood, left DLPFC functional deficits as measured by rCBF, are predicted by a symptom of decreased speech output but not by a diagnosis of schizophrenia or depression. The findings confirm our hypothesis that DLPFC dysfunction in the major psychoses is related to a behavioural deficit rather than a specific diagnosis. The data provide strong evidence that shared behavioural deficits in nosologically distinct psychiatric disorders may have common neurophysiological correlates.

In our study a single symptom, namely decreased speech output, was used to predict a neurophysiological deficit. A decrease in speech output in schizophrenia or depression is usually a manifestation of a more pervasive volitional disturbance that characterises psychomotor impairment. It can be argued that syndromes of psychomotor impairment, in depression and schizophrenia, represent disorders of willed or intentional behaviour. Intentional behaviour in this context may be defined as behaviour not extrinsically specified at the time of responding.

Previous studies have shown that depressed and schizophrenic patients display decreases in rCBF or regional metabolism in the left DLPFC. ${ }^{62-25}$ In separate studies of the depressed and schizophrenic patient samples, that comprise the data for this report, DLPFC dysfunction was significantly related to either a syndrome of psychomotor poverty or a syndrome of psychomotor retardation, respectively. ${ }^{89}$

Neither of these two groups displayed so called "hypofrontality", that is, a global decrease in frontal lobe perfusion when compared to controls. Both, however, displayed a focal decrease in prefrontal perfusion in the DLPFC. The present study extends these findings by demonstrating that DLPFC rCBF is predicted by a behavioural deficit rather than diagnosis. The findings also indicate that the inclusion of significant numbers of patients with psychomotor impairment, in studies of depression or schizophrenia, increases the probability of finding a decrease in DLPFC metabolism or perfusion. This association of DLPFC hypoperfusion with psychomotor impairment implies a three way link between abnormal DLPFC function in major psychiatric disorder, psychomotor impairment and the normal functional anatomy of the DLPFC. The evidence from this study is consistent with the proposal that the DLPFC plays an important part in the intrinsic generation of behaviour.

The possibility that our findings could be attributable to the confounding effects of medication needs consideration. A proportion of both patients' groupings (poverty and nonpoverty) were receiving either neuroleptics or antidepressant medication. In the case of antidepressants we have previously been unable to demonstrate significant effects on rCBF at thresholds of significance akin to those used in the present study. ${ }^{6} \mathrm{~A}$ small, but significant, effect on cerebral metabolism has been reported in patients receiving neuroleptics. ${ }^{26}$ However, the fact that a similar proportion of patients in the poverty and non-poverty groups were receiving neuroleptics makes a differential effect of this medication an insufficient explanation for the present findings.

A considerable number of animal and of human studies highlight a role for the DLPFC in intrinsic generation of behaviour. Lesion studies indicate a role in response selection in the absence of extrinsic information. ${ }^{27} \mathrm{~A}$ range of volitional and behavioural disturbances have been reported in human subjects with DLPFC lesions. These include a syndrome of decreased speech output, ${ }^{28}$ a reduction in both the frequency and variety of voluntary motility ${ }^{29}$ and more pervasive disturbances characterised by apathy, hypokinesis, lack of initiative and spontaneity. ${ }^{30}$ These clinical observations suggest that DLPFC lesions preferentially effect repertoires of behavioural expression that are independent of external guidance (ie, non-cued behaviour).

The functional specialisation of discrete brain systems can be established in studies of normal subjects using PET activation paradigms. The functional anatomy of intentional behaviour has been investigated in two separate studies, using a verbal fluency and a motor generation task, respectively. In both investigàtions the critical neuropsychological parameter was that of internal generation. Across both tasks the common region of activation was centred on the left DLPFC. ${ }^{31}$ These findings are of direct relevance to the present study as they highlight activations, during the performance of willed actions, in a cortical area that is dysfunctional in patients with poverty of speech. The findings could indicate that the fundamental deficit in patients with this symptom is that of internal generation. Furthermore, the 'largely asymmetric nature of the activations observed during tasks based upon internal generation agrees with the asymmetric findings in our study.

In the present study a decrease in rCBF was also seen in the poverty of speech group 
in the left angular gyrus. This cortical area is described as multimodal and its functions in humans include visuospatial orientation and attention. ${ }^{32-34}$ Previous studies have implicated this area in the psychomotor poverty syndrome of schizophrenia, of which poverty of speech is an important symptom. ${ }^{8}$ In a PET activation study, consisting of a motor generation task, activation of the DLPFC was associated with a decrease in $\mathrm{rCBF}$ in the left angular gyrus. ${ }^{31}$ This finding suggests that this cortical area may be part of a large scale neuronal network whose cooperative interactions with the DLPFC may be necessary in tasks involving internal generation.

Both schizophrenia and depression, as currently defined, encompass a wide range of phenomenological and neuropsychological deficits. In the absence of external biological discriminators the validity of the clinical distinction between these disorders has been questioned, particularly in the context of continuities in symptom profiles across both conditions. ${ }^{35}$ Therapeutic interventions have also failed to validate the distinction, with clinical response to pharmacological interventions being more strongly predicted by symptoms and not diagnosis. ${ }^{36}$ The findings of our study indicate an overlap in the neurobiological deficits of both depression and schizophrenia with a shared neurophysiological abnormality accounting for a similar symptom of poverty of speech.

Most biological studies of schizophrenia and depression are predicted on an assumption that both are distinct disease entities. Alternative viewpoints have posited a functional approach to psychopathology. ${ }^{37}$ The essence of this approach is that the basic units of classification in psychopathology are neither diseases nor syndromes but psychological dysfunctions. Within this framework the major psychoses, schizophrenia and depression, can be viewed as conglomerates of psychological dysfunctions that are nosologically nonspecific. This approach has echoes in more recent arguments for a symptom rather than a disease approach to psychiatric research. ${ }^{38}$ In the present study a symptom, but not a disease, was strongly predictive of a neurobiological dysfunction. This finding can be seen as supportive of a viewpoint that the study of symptoms, or symptom constellations, can provide information additional to that of categorical diagnostic systems in neurobiological studies of functional psychiatric disorders.

CJB and KF were both supported by the Wellcome Trust.

1 Edelman GM, Mountcastle VB. The Mindful Brain: cortical organisation and the group-selective theory of higher
brain function. Cambridge, MA: MIT Press, 1977: $7-100$.

2 Mountcastle VB, Lynch JC. Posterior parietal association cortex of the monkey: Command functions for operations within extrapersonal space. $\mathcal{f}$ Neurophysiol 1975 ; 38:871-909.

3 Buchsbaum MS, DeLisi LE, Holcomb HH, et al. Anteroposterior Gradients in Cerebral Glucose Use in Schizophrenia and Affective Disorders. Arch Gen Psychiatry 1984;41:1159-66.

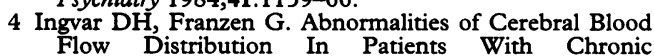

Schizophrenia. Acta Psychiatr Scand 1974;50:425-62.

5 Wolkin A, Angrist B, Wolf A, et al. Low Frontal Glucose Utilisation in Chronic Schizophrenia: A Replication Study. Am F Psychiatry 1988;145:251-3.

6 Bench CJ, Friston KJ, Brown RG, Scott L, Frackowiak RSJ, Dolan RJ. The Anatomy of Melancholia. Abnormalities of Regional Cerebral Blood Flow in Major Depression. Psychol Med 1992;22:607-15.

7 DeLisi LE, Holcomb HH, Cohen RM, et al. Positron Emission Tomography in Schizophrenic Patients With and Without Neuroleptic Medication. $f$ Cereb Blood Flow Metab 1985;5:201-6.

8 Liddle P, Friston KJ, Frith CD, Hirsch SR, Jones T, Frackowiak RSJ. Patterns of cerebral blood flow in schizophrenia. Br f Psychiatry 1992;160:179-86.

9 Bench CJ, Friston KJ, Brown R, Frackowiak RSJ, Dolan RJ. Regional cerebral blood flow (rCBF) in depression measured by positron emission tomography (PET): the relationship with clinical dimensions. Psychol Med 1993; 23:579-90.

10 Andreasen NC. The scale for assessment of negative symptoms. Iowa City: University of Iowa, 1983.

11 Liddle P. Schizophrenic syndromes, cognitive performance and neurological dysfunction. Psychol Med 1987;17:49-57.

12 Widlocher DJ. Psychomotor retardation: Clinical, theoretical, and psychometric aspects. Psychiatric Clin N Am 1983;6:27-40.

13 Endicott J, Spitzer RL. A Diagnostic Interview. The Schedule for Affective Disorders and Schizophrenia. Arch Gen Psychiatry 1978;35:837-44.

14 Spitzer RL, Endicott J, Robins E. Research diagnostic criteria for a selected group of functional disorders. New York: Biometrics Research Division, New York State Psychiatric Institute, 1977.

15 Hamilton M. A rating scale for depression. I Neurol Neurosurg Psychiatry 1960;23:56-62.

16 Hachinski VC, Iliff LD, Zilhka E, et al. Cerebral Blood Flow in Dementia. Arch Neurol 1975;32:632-7.

17 American Psychiatric Association. Diagnostic and statistical manual of mental disorders. Washington DC: American Psychiatric Association, 1980

18 Friston KJ, Frith CD, Liddle PF, Dolan RJ, Lammertsma AA, Frackowiak RSJ. The relationship between global and local changes in PET scans. 7 Cereb Blood Flow Metab 1990;10:458-66.

19 Friston KJ, Frith CD, Liddle PF, Frackowiak RSJ. Plastic Transformation of PET Images. 7 Comp Ass Tomog 1991;15:634-9.

20 Talairach J, Tournoux P. Co-planar stereotaxic atlas of the human brain. Stuttgart: George Thieme Verlag, 1988: human 122 .

21 Friston $\mathrm{KJ}$, Frith CD, Liddle PF, Frackowiak RSJ. Comparing Functional (PET) Images: The Assessment of Significant Change. 7 Cereb Blood Flow Metab 1991;11:690-9.

22 Baxter LR, Schwartz JM, Phelps ME, et al. Reduction of Prefrontal Cortex Metabolism Common to Three Types of Depression. Arch Gen Psychiatry 1989;46:243-50.

23 Weinberger DR, Berman KF, Zec RF. Physiologic Dysfunction of Dorsolateral Prefrontal Cortex in Schizophrenia. 1. Regional Cerebral Blood Flow Evidence. Arch Gen Psychiatry 1986;43:114-24.

24 Martinot IL, Hardy P, Feline A, et al. Left Prefrontal Glucose Hypometabolism in the Depressed State: A Confirmation. Am f Psychiatry 1990;147:1313-7.

25 Liddle PF, Friston KJ, Hirsch SR, Frackowiak RSJ. Regional Cerebral Metabolic Activity in Chronic Schizophrenia. Schiz Res 1990;3:23-4.

26 Wik G, Wiesel FA, Sjogren I, Blomqvist G, Greitz T, Stone Elander $S$. Effects of sulpiride and chlorpromazine on regional cerebral glucose metabolism in schizophrenic patients as determined by positron emisschizophrenic patients as determined by positron emis-
sion tomography. Psychopharmacology 1989;97:309-18.

27 Goldman-Rakic PS. Circuitry of primate prefrontal cortex and regulation of behaviour by representational memory. In: Mountcastle VB, et al, eds. Handbook of memory. In: Mountcastle VB, et al, eds. Handbook of physiology-The nervous system
and Wilkins, 1987:373-417.

28 Luria AR. Traumatic aphasia. The Hague: Mouton, 1970.

29 Klages W. Frontale und diencephale antriebsschwache. Arch Psychiatr Z Neurol 1954;191:365-87.

30 Stuss DT, Benson DF. Frontal lobe lesion and behaviour. In: Localisation in neuropsychology. Academic Press, 1983

31 Frith CD, Friston KJ, Liddle PF, Frackowiak RSJ. Willed action and the prefrontal cortex in man: a study with PET. Proc R Soc Lond B 1991;244:241-6.

32 Mesulam M-M. The functional anatomy and hemispheric specialization for directed attention. Trends Neurosci 1983;6:384-7.

33 Heilman KM, Pandya DN, Geschwind N. Trimodal inattention following parietal lobe ablations. Trans Am Neurol Assoc 1970;95:259-61.

34 Lynch JC. The functional organization of the posterior parietal astex Behav Brain Sci 1980;3:485-99.

35 Kendell RE, Gourlay J. The clinical distinction between the affective psychoses and schizophrenia. $\mathrm{Br} f$ Psychiatry 1970;117:261-6.

36 Johnstone EC, Crow TJ, Frith CD, Owens DGC. The Northwick park "Functional' Psychosis study: diagnosis and treatment response. Lancet 1988;ii:119-24.

37 van Praag HM, Leijnse B. Neubewertung des syndroms. Psychiatr Neurol Neurochir 1965;68:50-66.

38 Costello CG. Research on symptoms versus research on syndromes. Br ₹ Psychiatry 1992;160:304-8.
syntello 